\title{
Childhood chiasmal gliomas: update on the fate of patients in the 1969 San Francisco Study
}

\author{
RICHARD K IMES AND WILLIAM F HOYT \\ From the Neuro-ophthalmology Unit, Departments of Neurological Surgery, Neurology, and Ophthalmology, \\ University of California, San Francisco, San Francisco, California, USA
}

SUMmARY We review the current status of 28 patients with chiasmal glioma who were originally reported on by Hoyt and Baghdassarian in 1969. The median period of follow-up was 20 years. Sixteen patients are now dead, five from chiasmal glioma and the rest from other causes. Four of the five deaths from chiasmal glioma occurred before 1969. The mortality rate in patients with neurofibromatosis and those without neurofibromatosis was essentially the same. Nine of 16 patients with neurofibromatosis are dead, two from chiasmal glioma, one from aspiration, and six from another tumour. Seven of 12 patients without neurofibromatosis are dead, three from chiasmal glioma, two from an operation on their tumour, and two from a medical illness. In general the quality of life of the surviving patients is good; none reported having a noticeable decrease in vision since 1969.

In the report of their series in 1969 Hoyt and Baghdassarian' reviewed the clinical course of 36 optic gliomas of childhood, 29 of which involved the chiasm. They stressed that, in the natural course and despite treatment of any form, these tumours appeared to have a self-limited pattern of growth and morbidity, and that the prognosis for a long survival with useful vision was good. Fifteen years have passed since that series was published. Because rational management of a childhood chiasmal glioma must be based on knowledge of its long-term behaviour, and of the generalised condition of neurofibromatosis with which it frequently is associated, we reviewed the current status of the patients in that study who had chiasmal involvement.

\section{Material and methods}

\section{PATIENTS}

Of the 29 patients with chiasmal glioma reported in $1969,{ }^{1} 28$ (12 male, 16 female) are included in this study. The remaining patient is excluded now because later evaluations cast doubt on the original diagnosis.

The diagnosis of chiasmal glioma had been established histologically in 16 of the patients in this series

Correspondence to Dr W F Hoyt, Room M-876, Department of Ophthalmology, University of California, San Francisco, San Francisco, CA 94143, USA. and was based on observations at craniotomy in four. The diagnosis was presumptive in eight patients, all of whom were children who had neurofibromatosis, loss of vision, optic canal enlargement, and optic atrophy. Five of these children had an anteriorly situated mass in the third ventricle that involved the chiasm and one or both optic nerves, as shown by tomographic pneumoencephalography; two had sphenoid dysplasia and a J-shaped sella; and one had ambylopia and a temporal field defect.

\section{METHODS}

Follow-up data were obtained by direct contact with the patients, their parents, or their physician, or from hospital records. For patients who had survived the visual function was assessed on the basis of whether the patient could see well enough to read, drive, or recognise objects. Change in visual function was estimated by asking if vision had deteriorated since 1969. Quality of life was assessed subjectively on the basis of the patient's visual capacity, degree of independence, marital status, and level of education.

\section{Results}

The period of follow-up for the present report ranged from 0 to 44 years (median 20 years). The information obtained is current to July 1984, except in the 


\section{Living_patients}

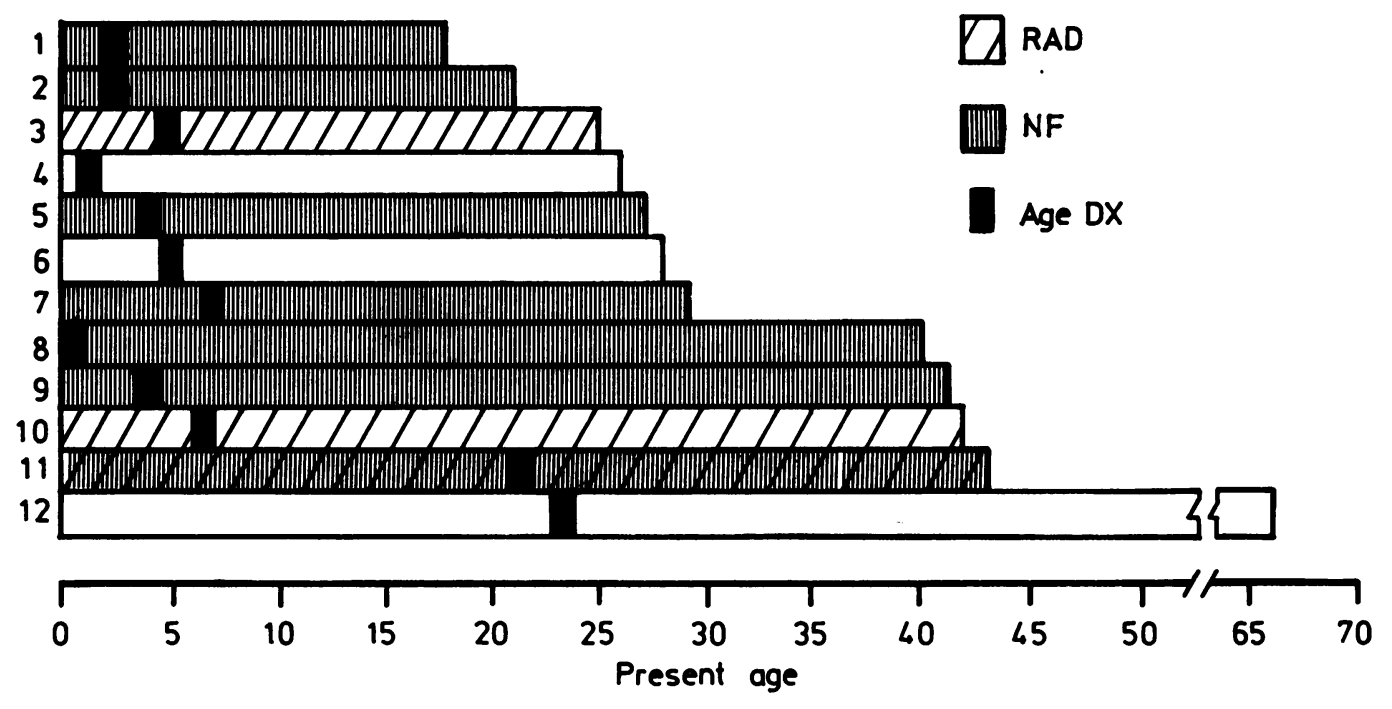

Deceased patients
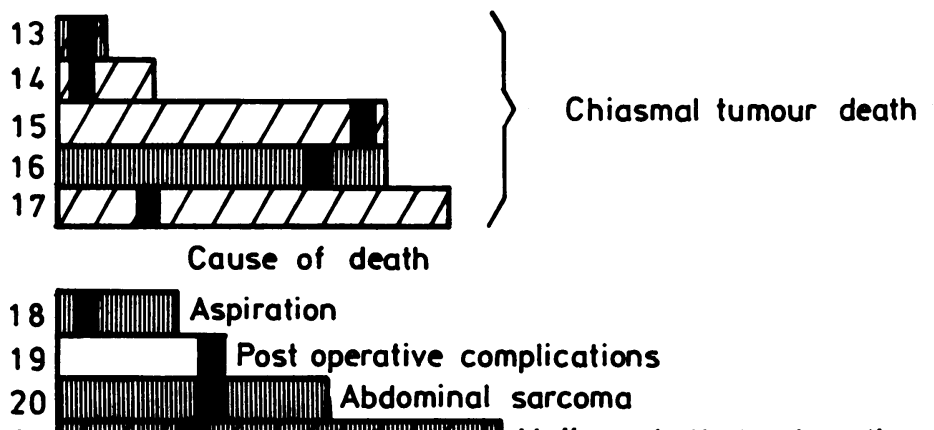

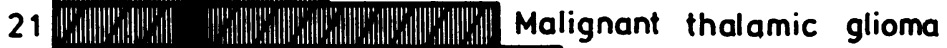

22 .

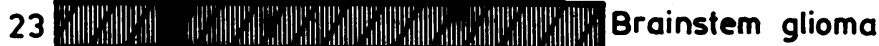

26 Will 25 171420 Operative complications

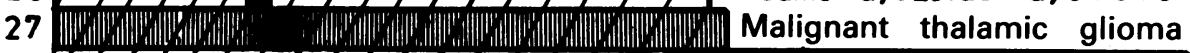

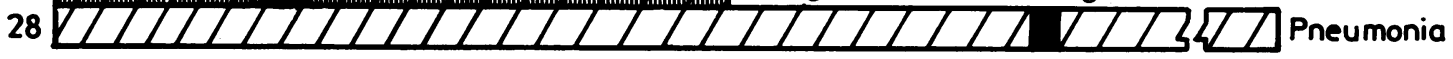

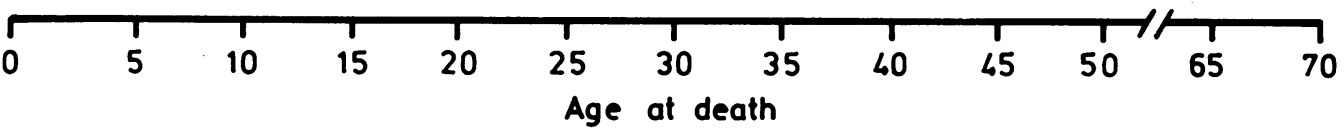

Fig. 1 Bar graph summarising data for the patients studied. Age of surviving patients is current to 1984, except for patients 1 and 7, for whom data were available only to 1975 and 1981 respectively.

case of patients 1 and 7 , for whom data were available only to 1975 and 1981 respectively.

Deaths. When this series originally was reported in
1969 , eight of the 28 patients were dead; eight more patients had died by the time of this review in 1984 (Fig. 1). The ages at death were distributed evenly 
between 2 and 31 years, except that one patient died at the age of 67. Five patients died of their chiasmal tumour; only one of these died after 1969. Most of the deaths in the series were from other causes.

Causes of death in patients with or without neurofibromatosis. Nine of the 16 deaths occurred in patients with neurofibromatosis. Two patients died from their chiasmal tumour at 2 and 15 years of age respectively. All deaths from non-chiasmal tumours occurred in this group: three patients died from sarcomatous degeneration of peripheral neurofibromas at 12,23 , and 26 years of age, and three others from a malignant glioma of the brain at 20 , 25 , and 31 years of age respectively. One patient aspirated fluid and died at age 5 years after removal of lingual and cervical neurofibromas.

Seven of the 16 deaths occurred in patients who did not have neurofibromatosis. Three of them died from chiasmal tumour at 4,15 , and 18 years of age, and two as a direct result of operations on their chiasmal tumour at 7 and 27 years of age respectively. Two died of medical illnesses: one of pneumonia, azotaemia, and chronic glomerulonephritis at 30 years of age, and one of pneumonia at age 67 years.

Causes of death in patients treated with or without radiation. Eleven of the 14 patients who received radiation therapy died, four from their chiasmal tumour at 2, 4, 15, and 18 years of age respectively, and the rest from other causes. Five of the 14 patients who did not receive radiation therapy died, one of her chiasmal tumour at 15 years of age and the rest from other causes.

Surviving patients. The 12 patients surviving at the time of this review ranged from 17 to 66 years of age; most were between 25 and 45 years old (Fig. 1). They have been followed up for 14 to 44 years (median follow-up 23 years). The diagnosis of chiasmal glioma had been established histologically in six of these patients, by observation at craniotomy in two, and by tomographic pneumoencephalography in four. Seven of the patients had neurofibromatosis. Three received radiation therapy.

In general these patients had a good quality of life. Five had married and were raising families; three were college graduates, one with a master's degree. Most were self-sufficient and could see well enough to read and/or drive, and most were employed. No patients reported that their vision had deteriorated noticeably since 1969 .

\section{Discussion}

This study represents the longest follow-up review of patients with chiasmal glioma yet reported. The 28 patients have been followed up for a median of 20 years since diagnosis. Sixteen of those patients
$(57 \%)$ are dead. Although this mortality rate is higher than that reported for other series, only five of our patients $(18 \%)$ died from their chiasmal glioma. Four of the deaths from chiasmal glioma occurred before 1969 , each within three years of diagnosis; the other occurred 14 years after diagnosis. This result suggests that the risk of death from a chiasmal glioma is greatest during the early follow-up period. Such a possibility is reflected also in the findings of Rush $e t$ $a l{ }^{2}$ Calculating the probability of survival in their 52 patients with chiasmal glioma, Rush et al. ${ }^{2}$ showed that the greatest risk of death was in the first decade after diagnosis. The 27 deaths from chiasmal glioma in their series occurred a median of four years after diagnosis, and their 23 surviving patients were alive a median of 17 years after diagnosis.

Our data do not support the suggestion made by Stern et al. ${ }^{3}$ in 1979 that optic glioma in patients with neurofibromatosis occurs preferentially in the optic nerve without involvement of the chiasm. Sixteen of our patients $(57 \%)$ had neurofibromatosis. Among the other reported series of patients with optic chiasmal glioma, eight of 18 patients $(44 \%)$ reported by Oxenhandler and Sayers, ${ }^{4} 14$ of 32 patients $(44 \%)^{*}$ reported by Lloyd, ${ }^{5} 15$ of 38 patients $(39 \%)^{*}$ reported by Miller et al., ${ }^{6} 10$ of 26 patients (38\%) reported by DeSousa et al., ${ }^{7}$ and six of 18 patients (33\%) reported by Danoff et al. ${ }^{8}$ had neurofibromatosis. Nor do our results support the notion that the presence of neurofibromatosis has a protective influence on survival, as was suggested by Rush et al. ${ }^{2}$ The mortality rate for our patients who had neurofibromatosis $(9 / 16$, or $56 \%)$ was essentially the same as that for our patients who did not $(7 / 12$, or $58 \%)$.

It appears in fact that such patients with neurofibromatosis may be at a greater risk of death from a non-chiasmal tumour than from their chiasmal glioma. Of our nine patients with neurofibromatosis who died only two died from their chiasmal glioma, whereas six died from other tumours-most within the second or third decade of life (Fig. 1). Moreover, all the deaths from a second tumour in our series were of patients with neurofibromatosis. There are several other reports of death from other tumours in children who had the combination of neurofibromatosis and chiasmal glioma: one child died of fibrosarcoma of the temporal lobe five years after discovery of the chiasmal glioma, ${ }^{8}$ one of a malignant peripheral nerve tumour 13 years after chiasmal glioma was diagnosed, ${ }^{4}$ and one of a cerebellar astrocytoma eight years after diagnosis of the chiasmal tumour. ${ }^{2}$

While many of our patients initially had a significant degree of visual impairment, their visual function has not changed appreciably since diagnosis.

* Our calculation from data provided in the article. 
Rush et $a .^{2}{ }^{2}$ reported essentially no change in the visual acuity in the better eye of their 52 patients with chiasmal glioma from the initial to the final examination; Wong and Lubow' ${ }^{9}$ reported that, except for patients who underwent a surgical procedure, their patients' vision remained relatively stable during the follow-up period. Glaser $e t$ al.,${ }^{10}$ reviewing 20 of the patients from our series in 1971, noted a monotonously stable course of field defects and visual acuity in most patients; in addition they found no perimetric evidence to support the efficacy of irradiation in those patients. All of these findings corroborate Hoyt and Baghdassarian's' earlier suggestions that visual impairment present at the time of diagnosis does not change appreciably thereafter.

Our review shows that nine of the 12 survivors in this series had no radiation therapy, whereas 11 of 16 patients who died, including four of the five who died from their chiasmal tumour, had undergone irradiation. Our series, however, affords no conclusive answers regarding the benefits or hazards of radiation therapy for these tumours. The two deaths after neurosurgical procedures in our series occurred in the immediate postoperative period; one early in the series was from hypothalamic complications and the other late in the series was the result of a carotid artery injury. We believe that both deaths were avoidable.

Except for the hypothalamic effects on growth and body habitus most of our surviving patients are normal, healthy, functioning members of society. We find, however, that the prognosis for survival in patients with chiasmal glioma is not as good as it was thought to be in 1969. For such patients with neurofibromatosis the poorer prognosis is not a factor of the chiasmal glioma, but of the potential for development of a malignant tumour elsewhere in the brain or in the peripheral nerves.

\section{References}

1 Hoyt WF, Baghdassarian SA. Optic glioma of childhood: natural history and rationale for conservative management. $\mathrm{Br} J$ Ophthalmol 1969; 53: 793-8.

2 Rush JA, Younge BR, Campbell RJ, MacCarty CS. Optic glioma. Ophthalmology (Rochester) 1982; 89: 1213-9.

3 Stern J, DiGiacinto GV, Housepian EM. Neurofibromatosis and optic glioma: clinical and morphological correlations. Neurosurgery $1979 ; 4: 524-8$.

4 Oxenhandler DC, Sayers MP. The dilemma of childhood optic gliomas. J Neurosurg 1978; 48: 34-41.

5 Lloyd LA. Gliomas of the optic nerve and chiasm in childhood. Trans Am Ophthalmol Soc 1973; 71: 488-535.

6 Miller NR, Iliff WJ, Green WR. Evaluation and management of gliomas of the anterior visual pathways. Brain 1974; 97: 743-54.

7 DeSousa AL, Kalsbeck JE, Mealey J, Ellis FD, Muller J. Optic chiasmatic glioma in children. Am J Ophthalmol 1979; 87: 376-81.

8 Danoff BF, Kramer S, Thompson N. The radiotherapeutic management of optic nerve gliomas in children. Int $J$ Radiat Oncol Biol Phys 1980; 6: 45-50.

9 Wong IG, Lubow M. Management of optic glioma of childhood: a review of 42 cases. In: Smith JL, ed. Neuro-ophthalmology. St Louis: Mosby, 1972; 6: 51-60.

10 Glaser JS, Hoyt WF, Corbett J. Visual morbidity with chiasmal glioma. Arch Ophthalmol 1971; 85: 3-12.

Accepted for publication 12 July 1985. 